\title{
Effect of Reducing Phytic Acid from Sudanese Sorghum Bicolor (F.G) Using Simple Technique Methods on Broilers Performance
}

\author{
Omar Eltyeb Omara Ahmed, Dr. Mohamed Eltigani Salih, Dr. Kamal Abdelbagi Mohammed \\ Faculty of Agriculture Studies, Graduate Collage, Sudan University Of Sciences and Technology \\ *Corresponding Author: Dr. Omar Eltyeb Omara Ahmed, Sudan University of Sciences and Technology, \\ Graduate Collage, Faculty of Agriculture Studies, Sudan
}

\begin{abstract}
Two experiments were conducted to investigate the effect of reducing sorghum phytate on broilers performance. Firstly, an Experiment was conducted to reduce phytic acid from Sudanese Sorghum bicolor (local name: Feterita Gadarif (F.G)) using simple technique methods. Approximate Analyses was done to determine the initial and final nutrition values of the grains while using processing methods and to measure the change of nutrient content after processing treatments. The treatments of processing were divided into five methods of process in addition of control (unprocessed cereal) such as dehulling, germination, soaking, vitamin $C$ and storing methods respectively, to reduce phytic acid content of the grains. The treatments shown significant decrease $(p>0.05)$ in phytic acid contents of the processed sorghum as compared with unprocessed one (control). The second experiment was divided into two periods: starter and finishing period. 300 old unsexed broilers (HybroB) were used in one day. Six experiment diets were formulated with different level of processed sorghum (F.G), with 5 replicates in complete randomized design. Feed intake, body weight were weekly recorded. Then weight gain and feed conversion ratio was calculated, mortality was also recorded. 26 birds from each treatment were randomly selected, weighted and slaughtered for deterring carcass dressing percentage. Internal organs weighted and serum was analyzed, Result obtained shows that feeding processed grain (F.G) to broilers has significantly affected ( $p>0.05)$ feed intake, body weight gain, dressing percentage, abdominal fat, live and hot weight. Treatments had a significant increase effect $(p>0.05)$ on whole blood serum contents, high level of cholesterol, glucose, calcium level, meat protein and fat contents were observed in birds which were fed on processed grains. Treatments had a significant increase (in p>0.01) in level of tibia ash, observed by birds fed on processed grains as compared with control.
\end{abstract}

Keywords: phytic acid, determination, processing methods, broiler performance

\section{INTRODUCTION}

Phytic acid is known as inositol hexakis phosphate (IP6) inositol poly phosphate, or phytate (when it is in salt form), discovered in 1903 (Mullaney 2012). Phytic acid has been termed as anti-nutrient due to its ability to bind minerals protein and starches, either directly or indirectly and thus alter their solubility functionality and absorption (Nelson, 1967) The effect of phytic acid inhuman and animals nutrition are related to the interaction of phytic acid with protein, vitamins and several minerals, thereby restricting their bio-extractability (Svanberg and Lorri, 1997). Lower serum lipids have been associated with higher intake of the phytic acid, and an addition of phytic acid to high cholesterol diet reduced both serum cholesterol and triacylglycarols. In broilers (P.A) showed lower feed consumption, severely reduced growth and leg problems as a sign of P-deficiency. In view of the anti nutritional effects of phytic acid many attempts have been made to eliminate phytic acid from foods by several methods such as soaking ,fermentation , storing, cooking, germination, dehulling (Sathe2002), (Mahgoub and Elhag1998), (Ockenden and Lott1997 ,Omara2000). In Sudan poultry diets content depend on sorghum bicolor especially (F.G), the cereals have a large amount of phytic acid (more than $887 \mathrm{mg} / 100 \mathrm{~g}$ ), and thus maybe alter the performance of poultry ,so the trial was conducted to use simple processing methods such as dehulling - soaking -germination -vitamin $\mathrm{C}$, and storing methods to reduce phytic acid compound from sorghum bicolor (F.G), the main dish in broiler diet in Sudan and to investigate the effect of that on Broiler performance, and to shed light on phytic acid as a controversial component. 


\section{Material AND Methods}

\subsection{Reducing of Sorghum Phytate Using Simple Technique Methods}

In the first trial of Sudanese Sorghum, Sorghum bicolor (Fatareta Gadarif) (F.G) was purchased from Gadarif State local market of Dura. $20 \mathrm{~kg}$ from the experimental sorghum was cleaned from damaged seeds and foreign objects. Then subjected separately to five treatments for processing using a simple technique to reduced phytic acid. Such as milling, soaking, germination, vitamin $\mathrm{C}$ and storing in addition to control (UN processed sorghum). Chemical composition of sorghum was analyzed before and after processing treatment to determine nutrients content and phytic acid in the grain

\subsection{Chemical Composition of Unprocessed Sorghum (F G )}

The seeds were cleaned manually to remove broken seeds, dust and other extraneous materials. The cleaned grains were milled into fine flour with hammer mill (Gibbons Electric, Essex K) to pass $0.4 \mathrm{~mm}$ mesh size screen and were stored at $4 \mathrm{C}$ before being used for their analysis. The seeds were chemically analyzed according to procedure of AOAC (1980). The sorghum protein content was determined by adopting standard AOAC (1995) method. Energy was calculated as described by Osbonrne and Voogt (1978). Minerals were determined in the sample by the dry-aching methods described by Chapman and Pratt (1961). The amount of iron was determined using atomic absorption spectroscopy (Perkin-Elmer 2380). Ammonium vanadate method of Chapman and Pratt (1982) Calcium was determined by a titration method described by Chapman and Pratt (1961), hydrochloric acid extractability of minerals was performed according to the Chapman and Mahjan (1988)

\subsection{Phytic Acid Determination}

Phytic acid was determined by the method described by Wheeler and Ferrel (1971) using $2.0 \mathrm{~g}$ dried sample.

\subsection{Processing Treatment}

\subsubsection{Dehuling}

$5.0 \mathrm{~kg}$ of cleaned cereals moistened by adding water before hulling, to soften the surface of the grain and facilitating detachment of the pressure inside the machine. The commercial machine combines two stages, dehulling and milling, the hulling grains were milled into fine flour then stored at $4 \mathrm{C}$ before being used for their analysis.

\subsubsection{Soaking}

$5 \mathrm{~kg}$ of the whole cleaned seeds were put in a pot filled with tap water. The grains were removed from the water after 12 hours then were sun -dried, then milled into fine flour with hammer mill (Gibbons Electric, Essex K) to pass $0.4 \mathrm{~mm}$ mesh size screen and were stored at $4^{\circ} \mathrm{C}$ before being used for their analysis. The processed seed flour was chemically analyzed according to procedure of AOAC (1980).

\subsubsection{Germination}

According to the method of Koua Kou et al (2008), $5 \mathrm{~kg}$ of the whole cleaned seeds were immersed in water overnight .The grains were spread on trays lined with cloth and were kept wet by frequent spraying water. After 96 hours, germinated grains were removed from the trays, sun -dried, then milled into fine flour with hammer mill (Gibbons Electric, Essex $\mathrm{K}$ ) to pass $0.4 \mathrm{~mm}$ mesh size screen and were stored at $4^{\circ} \mathrm{C}$ before being used for their analysis

\subsubsection{Vitamin $C$}

Added $1 \mathrm{~kg}$ of the whole cleaned seeds was milled in a laboratory mill to obtain fine flour, $150 \mathrm{mg}$ of ascorbic acid was mixed well with the sample and then the processed seed flour was chemically analyzed according to procedure of AOAC (1980). Phytic acid was determined by the method described by Wheeler and Ferrel (1971) using $2.0 \mathrm{~g}$ dried sample...then $2 \mathrm{ml}$ of the milled and dried sample was weighted to determined phytic acid.

\subsubsection{Storing Sorghum}

Cereal (fetareta gadarif) was stored for 12 month, $2 \mathrm{~kg}$ of the seeds then milled into fine flour with hammer mill (Gibbons Electric, Essex $\mathrm{K}$ ) to pass $0.4 \mathrm{~mm}$ mesh size screen and was stored at $4^{\circ} \mathrm{C}$ before being used for their analysis . 


\subsection{Statistical Analysis}

Each sample was analyzed in triplicate and the values were then averaged. Data was assessed by the analysis of variance (ANOVA) as described by Snedecor, Cochran (1987) and by Duncan-multiple range test at a probability of $\mathrm{p}<0.05$.

\subsection{Second Experiment: the Effect of Reducing Sorghum phytate on Broiler Performance}

\subsubsection{Experiment site and duration}

The experiment was carried out in full span-sided deep litter house, the house was located in east-west direction with flour walls, the house was cleaned, disinfected, bedding of saw. Each pen was provided with a feeder and drinker. The light was maintained for 24 Hours.

\subsubsection{Experimental Diets}

The cereal of the diet was treated by using five simple methods in addition of control (unprocessed grains), sex experimental diets were formulated approximately isocaloric, isonitrogenous to meet the requirement for broiler chicks as outline by NRC (1984), then chemical analysis was charred out for the experimental diets

\subsubsection{Experimental Birds}

A total of three hundreds unsexed, day old broiler chicks (Hybro B) were weighted and allotted randomly into 30 pens, in groups of 10 chicks per pen and 5 pen per treatment in a completely randomized design. The initial body weight of all chicks in each pen were adjusted to be approximately the same

\subsubsection{Management and Data Collection}

The experiment was divided into two periods. The first period (starting period) from 1-28 days old and the second period (finishing period) from 28 to 49 days old. The experiment diets were randomly assigned to pen, and a number of 5 pens for each treatment as replicates ( 5 replicate/treatment) Feed and water provided ad libitum. In the starting period bodyweight, feed intake was weekly recorded .Weight gain and feed conversion ratio (F.C.R) was calculated for the individual replicate of each dietary treatment. Mortality was reported as occurred and clinical signs were observed and reported. In the finishing period body weight, feed intake were weekly recorded .Weight gain and feed conversion ratio ( F.C.R ) were calculated for the individual replicate of the each dietary treatment Mortality was reported as occurred .Leg abnormalities were determined by subjective evaluation of each birds . Only chicks showing a medium or severe degree of bowing were considered to be abnormal. At the termination of the experiment birds were fasted over night to empty their digestive tract and to reduce the chance of carcass contamination during cleaning of ingested matter from the digestive tract. Twenty five chicks were randomly selected from each dietary treatment (5 birds / replicate), leg -banded individually weighted and slaughtered .Blood samples were clot and sera were collected in to clean tubes and allowed to clot and sera were separated by centrifugation at $3000 \mathrm{r} . \mathrm{p} \mathrm{m}$ for 5 minutes and stored at $20 \mathrm{c}$ until analyzed. The birds were scaled in a pot of boiling water and feather was plucked manually. The carcasses were washed and allowed to drain and eviscerated by ventral cut. The blisters were determined for each bird by breast, and each of chicks was given a score representing the number of blisters. Carcass and abdominal fat were weighted immediately .Dressing out percentage on carcass hot basis was calculated by expressing hot carcass weight to life weight. The carcasses were dissected into meat and bone for determination of meat to bone ratio in the breast and legs

\subsubsection{Chemical Methods}

The level of serum lipids was measured by procedure of Frings et al (1970). The serum calcium level was determined as described by Trinder (1960), calorimetric micro determination of calcium serum cholesterol was measured using the method of Kim and Goldbig (1969) Serum in organic phosphorous level was determined by using akit (Randox Laboratories U. K). The meat of carcass was thawed in a refrigerator for 24 hours. Then, approximately analyzed on dry matter basis for chemical components according to AOAC (1980). 
Effect of Reducing Phytic Acid from Sudanese Sorghum Bicolor (F.G) Using Simple Technique Methods on Broilers Performance

\subsubsection{Experimental Design and Statistical Analysis}

A complete randomized design was used and the data generated from the experiment were statistically analyzed by analysis of variance as outline by Steel and Torrie (1960). Duncan s multiple range test was used to determine level of significant between treatment means at $5 \%$ level of probability. (Thomas et al, 1942).

\section{RESULTS OF FIRST TRIAL}

\subsection{Results of Approximate Analysis, Minerals and Calculated energy of the Sorghum (F. G)}

Result is presented in table (1) and (2). The result indicated that sorghum (F. G) had a high percentage of crude protein, Ether Extract and metabolizable energy.

Table1: Chemical composition of sorghum (F.G) \%

\begin{tabular}{|l|l|}
\hline \multicolumn{1}{|c|}{ Chemical Profile } & \multicolumn{1}{c|}{ Sorghum(F. G) } \\
\hline Dry Matter & 93.83 \\
\hline Crude Protein & 14.3 \\
\hline Ether Extract & 4.58 \\
\hline Crude Fiber & 2.69 \\
\hline Nitrogen Free Extracts & 7.31 \\
\hline Metabolizable Energy $(\mathrm{Kcal} / \mathrm{Kg})$ & 383 \\
\hline Methionin & $8.5 \mathrm{mg} / 100 \mathrm{~g}$ \\
\hline Thiamin & $0.38 \mathrm{mg} / 100 \mathrm{~g}$ \\
\hline Lysine & $117.6 \mathrm{mg} / 100 \mathrm{~g}$ \\
\hline Niacin & $3.8 \mathrm{mg} / 100 \mathrm{~g}$ \\
\hline
\end{tabular}

-Analyzed values are means of duplicate sample. ME is calculated value by the equation of Carpenter and Clegg (1966).-NFE is calculated value.

Table2: Minerals content of sorghum bicolor (F.G) $\mathrm{mg} / 100 \mathrm{~g}$

\begin{tabular}{|l|l|}
\hline Chemical Profile & Sorghum (F. G) \\
\hline $\mathrm{Ca}$ & 12.8 \\
\hline $\mathrm{P}$ & 356 \\
\hline $\mathrm{Mg}$ & 103 \\
\hline $\mathrm{Fe}$ & 4.8 \\
\hline $\mathrm{Mn}$ & 122 \\
\hline
\end{tabular}

-Analyzed values are means of duplicate sample.

-ME is calculated value by the equation of Carpenter and Clegg (1966).

-NFE is calculated value.

\subsection{Chemical Composition of Sorghum Before and After Processing}

Shown in table (3), the result shown significant ( $p>0.05$ ) change in some nutrient value of processed grains in energy, $\mathrm{Ca}, \mathrm{P}$, and $\mathrm{Fe}$, and slight change in protein content.

Table3: Effect of treatments on sorghum chemical composition before and after processing

\begin{tabular}{|l|l|l|l|l|l|l|}
\hline Treatments & CP & Energy $(\mathrm{kcal} / \mathrm{kg})$ & $\mathrm{Ca}$ & $\mathrm{P}$ & Fe & Phytate \\
\hline Raw sorghum & 145.30 & $3100.50 \mathrm{~b}^{*}$ & $0.260 \mathrm{a}$ & $0.38 \mathrm{a}$ & $4.80 \mathrm{a}$ & $889.20 \mathrm{a}$ \\
\hline Dehulling & 143.00 & $3101.00 \mathrm{~b}$ & 0.252 & $0.30 \mathrm{~b}$ & $3.26 \mathrm{~b}$ & $445.06 \mathrm{~b}$ \\
\hline Germination & 143.4 & $3113.33 \mathrm{a}$ & $0.265 \mathrm{a}$ & $0.28 \mathrm{~b}$ & $3.26 \mathrm{~b}$ & $87.90 \mathrm{~g}$ \\
\hline Soaking & 145.00 & $3100.67 \mathrm{~b}$ & 0.250 & $0.28 \mathrm{~b}$ & $4.83 \mathrm{a}$ & $189.70 \mathrm{e}$ \\
\hline Vitamin C & 145.10 & $3100.00 \mathrm{~b}$ & 0.250 & $0.29 \mathrm{~b}$ & $4.90 \mathrm{a}$ & $122.40 \mathrm{f}$ \\
\hline Storing & 143.00 & $398.20 \mathrm{c}$ & 0.245 & $0.28 \mathrm{~b}$ & $3.30 \mathrm{~b}$ & $360.00 \mathrm{c}$ \\
\hline Overall mean & 144.13 & 2652.28 & 0.254 & $0.30 \mathrm{~b}$ & $4.06 \mathrm{a}$ & $336.94 \mathrm{~d}$ \\
\hline Standard Error $(\mathrm{SE} \pm)$ & 0.71 & 2.85 & 0.01 & 0.01 & 0.25 & 1.52 \\
\hline LSD $_{0.05}$ & $\mathrm{Ns}$ & 8.70 & Ns & 0.03 & 0.77 & 4.68 \\
\hline
\end{tabular}

$\mathrm{LSD}_{0.05}$ : Least significant difference at 0.05 (for mean separations and comparisons)

* Different small letters represent significant differences between treatments means in each column.

Mean squares (from analysis of variance (ANOVA) table) for effect of different treatments on sorghum chemical composition before and after processing 
Effect of Reducing Phytic Acid from Sudanese Sorghum Bicolor (F.G) Using Simple Technique Methods on Broilers Performance

\begin{tabular}{|c|c|c|c|c|c|c|c|}
\hline Source of variation & $\begin{array}{c}\text { Degree of } \\
\text { Freedom (F.D) }\end{array}$ & $\mathrm{CP}$ & Energy & $\mathrm{Ca}$ & $\mathrm{P}$ & $\mathrm{Fe}$ & Phytate \\
\hline Treatments & 5 & 3.6253 & 465.83 & 0.00016 & 0.0048 & 2.2189 & 293.0241 \\
\hline Experimental Error & 12 & 1.4961 & 24.339 & 0.000047 & 0.00022 & 0.1956 & 6.9239 \\
\hline F-calculated value & & 2.4230 & 19.14 & 3.53 & 21.45 & 11.58 & 42.753 \\
\hline Level of Significance & & Ns & $* * *$ & $*$ & $* * *$ & $* * *$ & $* * *$ \\
\hline $\begin{array}{c}\text { Coefficient of variation } \\
\text { (C.V. \%) }\end{array}$ & & 8.50 & 1.86 & 2.70 & 4.94 & 10.89 & 7.81 \\
\hline
\end{tabular}

n.s: not significant at 0.05

\subsection{Determination of Phytic Acid}

Results of determination of phytic acid in raw sorghum and processed sorghum (F.G) are present in table (4). The result indicated a significant decrease $(p>0.05)$ of phytic acid of all the processed treatments.

Table4: Effect of treatments on sorghum phytate $\mathrm{mg} / 100 \mathrm{~g}$ before and after processing

\begin{tabular}{|l|l|l|}
\hline \multicolumn{1}{|c|}{ Treatements } & \multicolumn{1}{c|}{ Phytic Acid contents } & \multicolumn{1}{c|}{ Loss \% } \\
\hline Raw Sorghum & $889.20 \mathrm{a}$ & 0 \\
\hline Dehulling & $445.20 \mathrm{~b}$ & 49.9 \\
\hline Germination & $87.90 \mathrm{~g}$ & 90.1 \\
\hline Soaking & $189.70 \mathrm{e}$ & 78.6 \\
\hline Vitamin C & $128.40 \mathrm{f}$ & 86.2 \\
\hline Storing & $360.00 \mathrm{c}$ & 59.6 \\
\hline
\end{tabular}

LSD 0.05: least significant difference at 0.05 level of significance (for mean separations and comparisons)

* Different small letters represent significant differences between treatments means in each column.

Composition of experimental diets along with their chemical analysis is presented in table (5) and table (6).

Table5: Composition of experimental diets (as fed percentages)

\begin{tabular}{|c|c|c|c|c|c|c|}
\hline \multicolumn{7}{|c|}{ Treatments } \\
\hline Parameters & $\begin{array}{c}\text { Unprocessed } \\
\text { sorghum } \\
\text { (control) }\end{array}$ & $\begin{array}{c}\text { Processed } \\
\text { sorghum } \\
\text { (dehulling) }\end{array}$ & $\begin{array}{c}\text { processed } \\
\text { sorghum } \\
\text { (germination ) }\end{array}$ & $\begin{array}{c}\text { processed } \\
\text { sorghum } \\
\text { (soaking ) }\end{array}$ & $\begin{array}{c}\text { processed } \\
\text { sorghum } \\
\text { (vitamin C) }\end{array}$ & $\begin{array}{c}\text { processed } \\
\text { sorghum } \\
\text { (storing ) }\end{array}$ \\
\hline Sorghum & 73 & 72 & 73 & 70 & 72 & 73.5 \\
\hline Ground nut meal & 13 & 13 & 13 & 16 & 14 & 12.5 \\
\hline Sesame meal & 5 & 5 & 5 & 5 & 5 & 5 \\
\hline Concentrate & 7 & 7 & 7 & 7 & 7 & 7 \\
\hline Oyster shell & 1 & 1 & 1 & 1 & 1 & 1 \\
\hline Salt & 0.5 & 0.5 & 0.5 & 0.5 & 0.5 & 0.5 \\
\hline
\end{tabular}

*super concentrate content $\%$ CP 46\%,fat2\%, Energy Kcal /Kg2300\%,lysin10.60, Methionine 2.49\%, Ca8\% ,Iron 200mg, Zinc 1000mg, Mg1400mg, Copper200mg ,Vita A200.00I.U ,Vitamin K 40mg, Vitamin B 100mg, Antibiotic 200mg, Antioxidant100mg

Table6: Calculated chemical analysis of experimental diets

\begin{tabular}{|l|c|c|c|l|l|l|}
\hline \multicolumn{7}{|c|}{ Treatments } \\
\hline Parameters & $\begin{array}{c}\text { Unprocessed } \\
\text { sorghum } \\
\text { (control) }\end{array}$ & $\begin{array}{c}\text { Processed } \\
\text { sorghum } \\
\text { (dehulling) }\end{array}$ & $\begin{array}{c}\text { processed } \\
\text { sorghum } \\
\text { (germination })\end{array}$ & $\begin{array}{c}\text { processed } \\
\text { sorghum ( } \\
\text { soaking })\end{array}$ & $\begin{array}{c}\text { processed } \\
\text { sorghum } \\
\text { (vitamin C) }\end{array}$ & $\begin{array}{c}\text { processed } \\
\text { sorghum } \\
\text { (storing })\end{array}$ \\
\hline MEK cal /kg & 3149 & 3090 & 3099 & 3153 & 3150 & 3088 \\
\hline $\begin{array}{l}\text { Crude } \\
\text { protein }\end{array}$ & 22.7 & 22.00 & 21.6 & 55.6 & 22.5 & 21.3 \\
\hline Lysine & 1.2 & 1.1 & 1.0 & 0.8 & 1.2 & 1.1 \\
\hline Methionine & 0.6 & 0.4 & 0.3 & 0.2 & 0.5 & 0.4 \\
\hline $\begin{array}{l}\text { Available } \\
\text { phosphorous }\end{array}$ & 0.7 & 0.5 & 0.4 & 0.2 & 0.4 & 0.3 \\
\hline Calcium & 1.2 & 1.0 & 0.9 & 0.5 & 0.5 & 1.0 \\
\hline
\end{tabular}


Effect of Reducing Phytic Acid from Sudanese Sorghum Bicolor (F.G) Using Simple Technique Methods on Broilers Performance

\begin{tabular}{|l|l|l|l|l|l|l|}
\hline Ether Extract & 5.2 & 4.2 & 5.0 & 4.0 & 5.2 & 4.5 \\
\hline Crude fiber & 5.6 & 5.2 & 5.5 & 6.1 & 5.6 & 5.7 \\
\hline
\end{tabular}

\subsection{Results of the Second Experiment}

\subsubsection{Production Performance of Broiler Chicks in Starting Period}

Table (7) shows the overall feed intake, final body weight gain and final feed conversion ratio in starter period, the result indicated no significant effect in feed intake and body weight gain. Treatment had slight increase on feed conversion ratio The feed conversion ratio tended to be high in birds fed processed seeds than those fed on unprocessed sorghum .None of the treatment had significant effect on mortality rate during the starting period of the experiment.

Table7: Overall performance of experimental chicks between (1-28) day old

\begin{tabular}{|c|l|l|l|l|l|l|}
\hline \multicolumn{7}{|c|}{ Treatments } \\
\hline Parameters & $\begin{array}{c}\text { Unprocessed } \\
\text { sorghum } \\
\text { (control) }\end{array}$ & $\begin{array}{c}\text { Processed } \\
\text { sorghum } \\
\text { (dehulling) }\end{array}$ & $\begin{array}{c}\text { processed } \\
\text { sorghum } \\
\text { (germination })\end{array}$ & $\begin{array}{c}\text { processed } \\
\text { sorghum } \\
\text { soaking }\end{array}$ & $\begin{array}{c}\text { processed } \\
\text { sorghum } \\
\text { (adding vita C) }\end{array}$ & $\begin{array}{c}\text { processed } \\
\text { sorghum } \\
\text { (storing })\end{array}$ \\
\hline $\begin{array}{c}\text { Feed intake } \\
\text { g/bird }\end{array}$ & 1753.80 & 1778.20 & 1763.20 & 1743.60 & 1772.80 & 1772.00 \\
\hline $\begin{array}{c}\text { Weight gain } \\
\text { g/bird }\end{array}$ & 850.26 & 847.78 & 849.30 & 849.36 & 850.16 & 850.34 \\
\hline F C R & 2.06 & 2.09 & 2.07 & 2.1 & 2.08 & 2.08 \\
\hline Mortality \% & 12 & 8 & 12 & 8 & 10 & 8 \\
\hline
\end{tabular}

**Means on the same row showing common superscripts are not significantly different at $0.05 \%$ level.

\subsubsection{Production performance of broiler chicks in finishing period}

Table (8) shows overall performance, feed intake, finally body weight gain and finally feed conversion ratio. The results revealed that dietary treatment had significant increase $(p<0.05)$ in feed intake and bird fed processed sorghum had significant high feed intake than birds fed unprocessed sorghum. Treatments had a significant increase $(\mathrm{p}<0.05)$ in over all weight gain. The weight gain tended to be statistically higher in birds fed processed sorghum. The highest body weight gain was registered in birds fed in germinated sorghum Fallowed by birds fed on sorghum treated by vitamin $\mathrm{C}$ and birds fed on stored sorghum respectively. Chicks fed on processed sorghum had slight increase on feed conversion ratio than those fed on unprocessed sorghum. None of the treatment had significant effect on mortality rate during of the finishing period of the experiment

Table8: Overall performance of the experimental chicks between (28 - 49) days old

\begin{tabular}{|l|l|l|l|l|l|l|l|}
\hline \multicolumn{7}{|c|}{ Treatments } \\
\hline $\begin{array}{l}\text { Paramet } \\
\text { ers }\end{array}$ & $\begin{array}{l}\text { Unprocessed } \\
\text { sorghum } \\
\text { (control) }\end{array}$ & $\begin{array}{c}\text { Processed } \\
\text { sorghum } \\
\text { (dehulling) }\end{array}$ & $\begin{array}{c}\text { processed } \\
\text { sorghum } \\
\text { (germination) }\end{array}$ & $\begin{array}{l}\text { processed } \\
\text { sorghum } \\
\text { (soaking) }\end{array}$ & $\begin{array}{l}\text { processed } \\
\text { sorghum } \\
\text { (vita C) }\end{array}$ & $\begin{array}{c}\text { processed } \\
\text { sorghum } \\
\text { (storing })\end{array}$ & $\begin{array}{c}\text { SE } \\
( \pm)\end{array}$ \\
\hline $\begin{array}{l}\text { Feed } \\
\text { intake } \\
\text { /bird }\end{array}$ & $\begin{array}{l}1939.56 \pm 32.58 \\
\mathrm{~b}\end{array}$ & $\begin{array}{l}1877.00 \pm 50.3 \\
7 \mathrm{~b}\end{array}$ & $2369.44 \pm 66.14 \mathrm{a}$ & $\begin{array}{l}1883.90 \pm 68.35 \\
\mathrm{a}\end{array}$ & $\begin{array}{l}2490.60 \pm 51 . \\
56 \mathrm{a}\end{array}$ & $\begin{array}{l}2015.36 \pm 53.86 \\
\mathrm{~b}\end{array}$ & 96.83 \\
\hline $\begin{array}{l}\text { Weight } \\
\text { gain } \\
\text { g/bird }\end{array}$ & $889.60 \pm 10.27 \mathrm{~d}$ & $\begin{array}{l}893.82 \pm 13.32 \\
\mathrm{~d}\end{array}$ & $1144.90 \pm 36.25 \mathrm{a}$ & $899.50 \pm 4.47 \mathrm{~d}$ & $\begin{array}{l}1093.50 \pm 60 . \\
55 \mathrm{~b}\end{array}$ & $1001.12 \pm 4.99 \mathrm{c}$ & 13.30 \\
\hline F C R & 2.01 & 2.09 & 2.06 & 2.09 & 2.1 & 2.01 & 10 \\
\hline $\begin{array}{l}\text { Mortalit } \\
\mathbf{y} \%\end{array}$ & 12 & 12 & 8 & 10 & 8 & -- \\
\hline
\end{tabular}

*Values are means of 5 replicate each of 10 birds

*Means On the same row showing common superscripts are not significantly different at $0.05 \%$ level.

* SE: stander error of the means

\subsubsection{Carcasses Performance}

The effect of feeding unprocessed sorghum on weight of broiler carcass and dressing out percentage is shown in table (9). The results revealed that feeding broiler processed seeds significantly increase the weight of hot carcasses. The results indicate that there is no significant effect $(p<0.05)$ on dressing out percentage. The treatment had significant effect $(\mathrm{p}<0.05)$ on carcasses cut as shown in Table $(10)$. The 
Effect of Reducing Phytic Acid from Sudanese Sorghum Bicolor (F.G) Using Simple Technique Methods on Broilers Performance

results indicate that feeding broiler processed sorghum significantly $(\mathrm{p}<0.05)$ increase the weight of breast, leg, wings, back and neck.

Table9: Effect of feeding processed sorghum on carcasses performance of broilers chicks (gm)

\begin{tabular}{|c|c|c|c|c|c|c|c|}
\hline \multicolumn{8}{|c|}{ Treatments } \\
\hline $\begin{array}{l}\text { Parameter } \\
\text { s }\end{array}$ & $\begin{array}{l}\text { Unprocesse } \\
\text { d sorghum } \\
\text { (control) }\end{array}$ & $\begin{array}{l}\text { Processed } \\
\text { sorghum } \\
\text { (milling) }\end{array}$ & $\begin{array}{l}\text { processed } \\
\text { sorghum } \\
\text { (germination ) }\end{array}$ & $\begin{array}{l}\text { processed } \\
\text { sorghum } \\
\text { soaking })\end{array}$ & $\begin{array}{l}\text { processed } \\
\text { sorghum( } \\
\text { vita C) }\end{array}$ & $\begin{array}{l}\text { Processed } \\
\text { sorghum } \\
\text { (storing ) }\end{array}$ & $\begin{array}{l}\text { SE } \\
( \pm)\end{array}$ \\
\hline $\begin{array}{l}\text { Live } \\
\text { weight }\end{array}$ & $\begin{array}{l}1783.20 \pm 3.5 \\
6 \mathrm{e}\end{array}$ & $\begin{array}{l}1783.80 \pm 2 \\
.59 \mathrm{e}\end{array}$ & $2018.40 \pm 22.35 a$ & $\begin{array}{l}1848.92 \pm 1.0 \\
5 \mathrm{~d}\end{array}$ & $\begin{array}{l}1993.46 \pm 1 \\
.46 \mathrm{~b}\end{array}$ & $\begin{array}{l}1890.8 \pm 2.7 \\
7 \mathrm{c}\end{array}$ & $\begin{array}{l}4.2 \\
0\end{array}$ \\
\hline Hot weight & $\begin{array}{l}1259.20 \pm 4.9 \\
7 \mathrm{e}\end{array}$ & $\begin{array}{l}1263.08 \pm 3 \\
.59 \mathrm{e}\end{array}$ & $1456.40 \pm 18.13 \mathrm{a}$ & $\begin{array}{l}1339.16 \pm 1.2 \\
5 \mathrm{~d}\end{array}$ & $\begin{array}{l}1432.45 \pm 1 \\
.85 b\end{array}$ & $\begin{array}{l}1368.66 \pm 3 \\
03 \mathrm{c}\end{array}$ & $\begin{array}{l}5.9 \\
3 \\
\end{array}$ \\
\hline $\begin{array}{l}\text { Dressing } \\
\%\end{array}$ & 70.6 & 70.8 & 72.1 & 72.4 & 71.8 & 72.3 & \\
\hline
\end{tabular}

*Means On the same row showing common superscripts are not significantly different at $0.05 \%$ level

* SE stander error of the means

Table10: Effect of processed sorghum on broiler carcass cut weight (gm)

\begin{tabular}{|c|c|c|c|c|c|c|c|}
\hline \multicolumn{8}{|c|}{ Treatments } \\
\hline Parameters & $\begin{array}{l}\text { Unproces } \\
\text { sed } \\
\text { sorghum } \\
\text { (control) }\end{array}$ & $\begin{array}{c}\text { Processed } \\
\text { sorghum } \\
\text { (dehulling) }\end{array}$ & $\begin{array}{c}\text { processed } \\
\text { sorghum } \\
\text { (germination } \\
\text { ) }\end{array}$ & $\begin{array}{c}\text { processed } \\
\text { sorghum } \\
\text { (soaking ) }\end{array}$ & $\begin{array}{l}\text { processe } \\
\text { d } \\
\text { sorghum } \\
\text { (vita C) }\end{array}$ & $\begin{array}{l}\text { processed } \\
\text { sorghum } \\
\text { (storing ) }\end{array}$ & $\begin{array}{l}\mathbf{S} \\
\mathbf{E} \\
( \pm \\
)\end{array}$ \\
\hline Breast weight & $\begin{array}{l}410.10 \\
\pm 1.84 \mathrm{c}\end{array}$ & $\begin{array}{l}408.60 \pm 4.2 \\
5 c\end{array}$ & $\begin{array}{l}474.0 \\
46.87 \mathrm{a}\end{array}$ & $\begin{array}{l}436.36 \quad \pm \\
077 b\end{array}$ & $\begin{array}{l}436.80 \pm \\
0.97 b\end{array}$ & $\begin{array}{l}477.76 \quad \pm \\
2.29 \mathrm{a}\end{array}$ & $\begin{array}{l}1 . \\
59\end{array}$ \\
\hline $\begin{array}{l}\text { Leg and thigh } \\
\text { weight }\end{array}$ & $\begin{array}{l}421.52 \pm 1 \\
46 \mathrm{e}\end{array}$ & $\begin{array}{l}422.06 \pm 2.4 \\
1 \mathrm{e}\end{array}$ & $\begin{array}{l}484.70 \\
6.51 \mathrm{a}\end{array}$ & $\begin{array}{l}447.02 \quad \pm \\
1.41 \mathrm{~d}\end{array}$ & $\begin{array}{l}477.92 \pm \\
0.79 \mathrm{~d}\end{array}$ & $\begin{array}{l}454.84 \\
\pm 3.29 \mathrm{c}\end{array}$ & $\begin{array}{l}1 . \\
46\end{array}$ \\
\hline Wings weight & $\begin{array}{l}160.10 \pm 0 . \\
68 \mathrm{c}\end{array}$ & $\begin{array}{l}161.56 \pm 1.4 \\
3 e^{-}\end{array}$ & $\begin{array}{l}186.88 \\
1.02 \mathrm{a}\end{array}$ & $\begin{array}{ll}170.16 \quad \pm \\
1.19 \mathrm{~d}\end{array}$ & $\begin{array}{l}181.60 \pm \\
1.25 \mathrm{~b}\end{array}$ & $\begin{array}{ll}172.76 \quad \pm \\
2.23 \mathrm{c}\end{array}$ & $\begin{array}{l}0 . \\
62\end{array}$ \\
\hline $\begin{array}{l}\text { Neck and back } \\
\text { weight }\end{array}$ & $\begin{array}{l}262.28 \pm 2 . \\
43 \mathrm{~d}\end{array}$ & $\begin{array}{l}263.38 \pm 1.3 \\
9 d\end{array}$ & $\begin{array}{l}301.92 \\
6.15 \mathrm{a}\end{array}$ & $\begin{array}{l}208.28 \quad \pm \\
0.74 \mathrm{a}\end{array}$ & $\begin{array}{l}295.00 \pm \\
3.69 \mathrm{~b}\end{array}$ & $\begin{array}{l}283.68 \quad \pm \\
2.59 \mathrm{c}\end{array}$ & $\begin{array}{l}1 . \\
46\end{array}$ \\
\hline
\end{tabular}

*Means on the same row showing common superscripts are not significantly different at $0.05 \%$ level.

* SE stander error

\subsubsection{Meat /Bone Ratio and Carcasses Section}

The effect of feeding processed sorghum on meat to bon ratio and weight of meat of breasts and leg is shown in Table (11). Birds fed on germinated sorghum Fallowed by birds fed on sorghum treated by vitamin $\mathrm{C}$, and birds fed on stored sorghum respectively had a significantly $(\mathrm{p}<0.05)$ increase on thigh and legs meat ,than those fed unprocessed sorghum. The proportions of meat to bone in the breast, drumstick and thigh cut were not significantly affected by treatment

Table11: Effect of feeding processed sorghum on broiler carcass section weight $(\mathrm{g} m)$ and meat to bone ratio

\begin{tabular}{|l|l|l|l|l|l|l|l|}
\hline \multicolumn{9}{|c|}{ Treatments } \\
\hline $\begin{array}{l}\text { Parame } \\
\text { ters }\end{array}$ & $\begin{array}{l}\text { Unprocesse } \\
\text { d sorghum } \\
\text { (control) }\end{array}$ & $\begin{array}{l}\text { Processed } \\
\text { sorghum } \\
\text { dehulling }\end{array}$ & $\begin{array}{l}\text { processed } \\
\text { sorghum } \\
\text { (germination })\end{array}$ & $\begin{array}{l}\text { processed } \\
\text { sorghum } \\
\text { soaking })\end{array}$ & $\begin{array}{l}\text { processed } \\
\text { sorghum } \\
\text { (adding vita C) }\end{array}$ & $\begin{array}{l}\text { processed } \\
\text { sorghum } \\
\text { (storing })\end{array}$ & $\begin{array}{l}\text { SE } \\
( \pm)\end{array}$ \\
\hline $\begin{array}{l}\text { Breast } \\
\text { meat }\end{array}$ & $\begin{array}{l}344.06 \pm \\
1.83 \mathrm{c}\end{array}$ & $\begin{array}{l}345.00 \quad \pm \\
1.23 \mathrm{c}\end{array}$ & $399.96 \pm 1.34 \mathrm{a}$ & $\begin{array}{l}365.66 \quad \pm \\
0.90 \mathrm{~b}\end{array}$ & $365.40 \pm 1.47 \mathrm{a}$ & $\begin{array}{l}400.04 \\
\pm 1.47 \mathrm{a}\end{array}$ & $\begin{array}{l}0.6 \\
2\end{array}$ \\
\hline $\begin{array}{l}\text { Meat } \\
\text { /bone }\end{array}$ & 5.80 & 5.81 & 6.70 & 6.23 & 6.21 & 6.76 & - \\
\hline $\begin{array}{l}\text { Leg and } \\
\text { thigh } \\
\text { meat }\end{array}$ & $\begin{array}{l}353.14 \pm \pm \\
1.28 \mathrm{~d}\end{array}$ & $\begin{array}{l}353.80 \pm \\
1.77 \mathrm{~d}\end{array}$ & $408.32 \pm 4.86 \mathrm{a}$ & $\begin{array}{l}373.76 \quad \pm \\
2.70 \mathrm{c}\end{array}$ & $400.32 \pm 1.34 \mathrm{a}$ & $\begin{array}{l}381.36 \\
\pm 2.34 \mathrm{~b}\end{array}$ & $\begin{array}{l}1.2 \\
0\end{array}$ \\
\hline $\begin{array}{l}\text { Meat/b } \\
\text { one }\end{array}$ & 4.70 & 4.71 & 5.52 & 5.01 & 5.40 & 5.11 & \\
\hline
\end{tabular}

*Means on the same row showing common superscripts are not significantly different at $0.05 \%$ level.

* SE; stander error of the means 
Effect of Reducing Phytic Acid from Sudanese Sorghum Bicolor (F.G) Using Simple Technique Methods on Broilers Performance

\subsubsection{Abdominal Fat}

The results of abdominal fat and abdominal fat percentage indicated that there was a significantly increase $(\mathrm{p}<0.05)$ by treatment as shown in Table $(12)$.

Table12: Effect of feeding processed sorghum on abdominal fat as a percentage of the live weight

\begin{tabular}{|c|l|l|l|l|l|l|}
\hline \multicolumn{7}{|c|}{ Treatments } \\
\hline Parameters & $\begin{array}{c}\text { Unprocessed } \\
\text { sorghum } \\
\text { (control) }\end{array}$ & $\begin{array}{c}\text { Processed } \\
\text { sorghum } \\
\text { (milling) }\end{array}$ & $\begin{array}{c}\text { processed } \\
\text { sorghum } \\
\text { (germination ) }\end{array}$ & $\begin{array}{c}\text { processed } \\
\text { sorghum ( } \\
\text { soaking ) }\end{array}$ & $\begin{array}{c}\text { processed } \\
\text { sorghum } \\
\text { (adding vita C) }\end{array}$ & $\begin{array}{c}\text { processed } \\
\text { sorghum } \\
\text { (storing ) }\end{array}$ \\
\hline $\begin{array}{c}\text { Abdominal } \\
\text { fat wt (gm) }\end{array}$ & 49.5 & 49.7 & 56.7 & 51.4 & 55.4 & 52.6 \\
\hline $\begin{array}{c}\text { Abdominal } \\
\text { fat \% }\end{array}$ & 2.7 & 2.8 & 3.6 & 4.0 & 2.9 & 2.8 \\
\hline
\end{tabular}

*Means on the same row showing common superscripts are not significantly different at $0.05 \%$ level.

* SE: stander error of the means

\subsubsection{Meat Composition}

The results of meat composition are shown in table (13). Results indicated that treatments had a significantly $(p<0.05)$ increase on meat protein and fat content, and the treatment had no significant effect on moisture content, moreover birds fed on processed seeds had slight increase on ash content than those fed on unprocessed seeds.

Table13: Effect of feeding processed sorghum on broiler meat chemical composition

\begin{tabular}{|l|l|l|l|l|l|l|}
\hline \multicolumn{7}{|c|}{ Treatments } \\
\hline $\begin{array}{l}\text { Paramete } \\
\text { rs }\end{array}$ & $\begin{array}{l}\text { Unprocesse } \\
\text { d sorghum } \\
\text { (control) }\end{array}$ & $\begin{array}{l}\text { Processed } \\
\text { sorghum } \\
\text { (milling) }\end{array}$ & $\begin{array}{l}\text { processed } \\
\text { sorghum } \\
\text { (germination })\end{array}$ & $\begin{array}{l}\text { processed } \\
\text { sorghum( } \\
\text { soaking })\end{array}$ & $\begin{array}{l}\text { processed } \\
\text { sorghum } \\
\text { (adding vita C) }\end{array}$ & $\begin{array}{l}\text { processed } \\
\text { sorghum } \\
\text { (storing })\end{array}$ \\
\hline Moisture & 71.1 & 70.2 & 69.8 & 70.7 & 70.3 & 70 \\
\hline C. P & 19.9 & 19.94 & 21.97 & 20.5 & 21.0 & 19.2 \\
\hline Fat & 2.0 & 2.2 & 2.5 & 3.0 & 3.1 & 3.2 \\
\hline Ash & 1.2 & 1.3 & 1.4 & 1.3 & 1.4 & 1.3 \\
\hline
\end{tabular}

\subsubsection{Serum Composition}

The effect of treatment on serum composition of broiler shown in Table (14). The results indicated a significantly $(\mathrm{p}<0.05)$ decrease on cholesterol content in whole blood serum, obtained by birds fed on germinated sorghum Fallowed by birds fed on sorghum treated by vitamin C, and birds fed on stored sorghum respectively, The result shown a significant increase $(p<0.05)$ in blood serum glucose obtained by bird fed processed seeds The result indicated a significant increase $(p<0.05)$ effect on calcium and phosphorous content in whole blood serum in birds fed on processed seeds .

Table14: Effect of feeding processed sorghum on serum composition of broiler chicks

\begin{tabular}{|c|c|c|c|c|c|c|c|}
\hline \multicolumn{8}{|c|}{ Treatments } \\
\hline Para & $\begin{array}{c}\text { Unprocessed } \\
\text { sorghum } \\
\text { (control) }\end{array}$ & $\begin{array}{l}\text { Processed } \\
\text { sorghum } \\
\text { (milling) }\end{array}$ & $\begin{array}{c}\text { processed } \\
\text { sorghum } \\
\text { (germination } \\
\text { ) }\end{array}$ & $\begin{array}{c}\text { processed } \\
\text { sorghum } \\
\text { ( soaking } \\
\text { ) }\end{array}$ & $\begin{array}{l}\text { processed } \\
\text { sorghum } \\
\text { (vita C) }\end{array}$ & $\begin{array}{l}\text { processed } \\
\text { sorghum } \\
\text { (storing ) }\end{array}$ & $\begin{array}{l}\text { SE } \\
( \pm)\end{array}$ \\
\hline $\begin{array}{c}\text { Cholesterol } \\
(\mathrm{mg} / \mathrm{dl})\end{array}$ & $134.14 \pm 0.86 a$ & $126.76 \pm 0.93 b$ & $\begin{array}{l}126.76 \\
2.57 \mathrm{c}\end{array}$ & $\begin{array}{l}121.12 \pm \\
\text { o72c }\end{array}$ & $\begin{array}{l}120.74 \pm \\
1.36 \mathrm{c}\end{array}$ & $\begin{array}{l}121.02 \pm \\
4.56 \mathrm{c}\end{array}$ & 1.02 \\
\hline $\begin{array}{c}\text { Glucose (mg } \\
\text { /dl ) }\end{array}$ & $176.16 \pm 2.28 b$ & $181.04 \pm 1.60 \mathrm{a}$ & $\begin{array}{l}181.98 \\
2.97 \mathrm{a}\end{array}$ & $\begin{array}{l}182.76 \quad \pm \\
3.27 \mathrm{a}\end{array}$ & $\begin{array}{l}181.54 \\
\pm 3.41 \mathrm{a}\end{array}$ & $\begin{array}{l}182.80 \quad \pm \\
2.69 \mathrm{a}\end{array}$ & 1.24 \\
\hline $\begin{array}{l}\text { Calcium } \\
\text { (mg/dl ) }\end{array}$ & $10.86 \pm 0.27 b$ & $11.54 \pm 0.65 \mathrm{a}$ & $11.92 \pm 0.31 \mathrm{a}$ & $\begin{array}{ll}11.78 \quad \pm \\
0.23 \mathrm{a} & \\
\end{array}$ & $\begin{array}{l}11.70 \\
\pm 0.40 \mathrm{a}\end{array}$ & $\begin{array}{ll}12.10 \quad \pm \\
1.73 \mathrm{a} & \end{array}$ & 0.18 \\
\hline $\begin{array}{l}\text { Phosphorous } \\
\text { ( mg /dl) }\end{array}$ & $7.27 \pm 0.39 a$ & $8.46 \pm 0.21 b$ & $\begin{array}{l}5.96 \pm 0.96 \pm \\
0 .\end{array}$ & $\begin{array}{ll}6.04 & \pm \\
0.23 \mathrm{c} & \end{array}$ & $\begin{array}{ll}6.04 & \pm \\
0.23 \mathrm{c} & \end{array}$ & $\begin{array}{ll}6.14 & \pm \\
0.25 b c & \end{array}$ & 0.12 \\
\hline
\end{tabular}

*Means on the same row showing common superscripts are not significantly different at $0.05 \%$ level.

* SE stander error of the means 


\section{DISCUSSION}

\subsection{Chemical Composition}

The moisture content of sorghum bicolor (F.G) was in the range obtained by AbdElnour (2001) who indicated that moisture content of Feterita and Dabar was 9.6-8.75 respectively. The crude protein content of sorghum bicolor (F.G ) ( Table 3 ) was in the range observed by Hulse et al (1980) who found that the protein content of sorghum bicolor ranged between 8-16\% ,but lower than Elsayed (1999) analyzed the protein content of Tabat and Fetarita was 6.46-9.11, but higher than the value reported by Dillon (2007), and lower than that obtained by Mayada (2009).The metabolizable energy of sorghum ( F.G ) was in the range detected by Idris (2004) who found the carbohydrates content for sorghum was $80.7 \%$. Minerals content was in range optimum by Hulse et al (1980) and Idris (2004)

\subsection{Phytic Acid in Unprocessed Grains}

The phytic acid content of sorghum (F.G ) ( Table4 ) are close to those reviewed by Marfo et al (1990) who reported that phytic acid content of red sorghum was 886mg /100g. Greiner (2006), and Konietzny and Koyode (2006) whom found that sorghum phytate ranged from 590 to 1180 and from 400 to $3500 \mathrm{mg} / 100 \mathrm{~g}$ dwt. The result was higher than the range obtained by Eammambux et al (2009) and higher than the range reported by Makokha et al (2002), also higher than the found reported by Sathe (2002), these can be explained by that phytic acid content varied, stage of maturity, climatic, conditions type of soil, amount of available phosphorous and milling fraction of the grains

\subsection{Removal of Phytate}

Table (4) shown the removal of phytate after processed treatments, the highest loss of phytate ( $>0.05$ ) obtained by germination method $(90.1 \%$ ) flowed by vitamin C method (86.2\%),soaking method (78.6\%),storing method (59.6\%) and dehulling method (49.9\%) respectively. In fact phytic acid in flour can be hydrolyzed by the enzyme phytase, and the optimum condition for phytase activity are $\mathrm{PH}$ range from 5.0 to 5.5 and temperature rang 50 to $55 \mathrm{c}$.Germination method in sorghum grains reduced phytate up t0 90.9 after $96 \mathrm{~h}$ Wisal (2004). The obtained was on line with Abdelrahman et al (2007) who reported that germination increase part of both major and trace minerals and also reduced significantly the phytic acid, germination is more effective way to remove phytic acid, and germination in $80-90 £$ removed $92 \%$ of phytate and releases vitamins and make grains and seeds more digestible. Vitamin C method reduced (P.A) $86.2 \%$, vita C is strong enhancer of plant iron can overcome the inhibitors in plant foods. One study found that various doses of phytate reduced iron absorption by 10 to $50 \%$.But adding $50 \mathrm{mg}$ of vitamin $\mathrm{C}$ counteracted the phytate and adding $150 \mathrm{mg}$ of vitamin $\mathrm{C}$ increased iron absorption to almost $30 \%$, similarly, in the presence of a large dose of tannic acid, $100 \mathrm{mg}$ of vitamin $\mathrm{C}$ increased iron absorption from 2-8\%. Snedecor et al (1987), this could be attributed to the fact that acidic anion complexes with the minerals like Calcium and Phosphorus result in an improvement in the digestibility DF these minerals as reported by several workers. The results of soaked sorghum was in range of result obtained by Aotzc and Others, , 2001) who reported that soaking of maize for $1 \mathrm{~h}$ at room temperature already led to be reduction of phytic acid by $51 \%$, but lower than the report obtained by Mahgoub and Elhage (1998) whom reported that soaking of sorghum flour at room temperature for $24 \mathrm{~h}$ reducing phytic acid level by $16-21 \%$. The reduced of phytic acid using storing method was present in table ( 4 ) the result was in the same line with Sathe (2002), who reported that the decreased of phytic acid during storage from 0 to $65 \%$ in cereal according to temperature and humidity and from 2.5 to $76 \%$ in legume, and the reduction depend on the type of seeds, storage condition, and the age of the seeds. The processed of sorghum bicolor (F.G) by using five technical methods changed the nutrient values of the seeds after processing is shown in Table (3). The high content of crude protein metabolizable energy and fat obtained by germinated seeds, the result on line with many workers, they observed increase in proteins during germination of cereals, this increase could be attributed to a synthesis of enzymatic proteins by germination seeds . (W H O, 1998) Marero et al (1988) also reported that the increases in protein might be due to the fact some amino acids are produced in excess of the requirement during protein synthesis and these tend to accumulate in free amino acids pool. In the same line Koua kou et al (2008) indicated that the seeds of cereals during their germination develop a strong enzymatic activity. The results also in line with Ocheme and Chinma , (2007) who find that germination 
significantly increases the protein dry mater and ash content, while fat content and energy values of the flour sample showed a decrease , these protein increase not exceeding $14 \%$ of the starting protein content . This was found to be attributed to loss of dry weight through respiration during germination, in the same line Beal and Mehta (1985) reported that germination reducing up to $75 \%$ of phytate and increase phytase activity, but different from Martinez et al ( 1980 ) who reported that germination decreased the content of lysine and tryptophan and vitamin such as C, B, A and E.

\subsection{Production Performance of the Experimental Chicks}

As expected the treatments of processed sorghum was led to greater performance. Performance indices such as body weight, body weight gain. Feed efficiency was higher with processed sorghum diets than unprocessed sorghum diets. Body weight gain differed significantly in the various treatments, the higher body weight gain obtained by birds fed on germinated sorghum fallowed by birds fed on processed sorghum with vitamin $\mathrm{C}$ and birds fed on stored sorghum, soaked and dehulling sorghum respectively. The great increase of body weight gain synchronization inversely with the decreased of sorghum phytate. These can be explained by the finding of Doherty et al (1982) who reported that phytic acid reduced the growth. The results was on line with Harland (1936) who indicated that growth and zinc utilization was inhibited by phytic acid in rats and human, phytic acid interferes with enzymes need to digest food, including pepsin, which is needed for breakdown of protein in the stomach and amylase, which is required for the breakdown of starch, and inhibits the enzyme trypsin which is needed for protein digestion in small intestine .The high feed intake obtained by birds fed on germinated seeds was explained by the find of Mamudu et al (2005)who reported that germination is causing profound changes in the seeds and derivatives, thus giving them especial flavor, on account of hydrolysis of starch ,derived porridge from cereal which has a low viscosity ,thus the porridge from cereal sprouts for children have a nutritional certain advantage: high energy density intake of macronutrients and micro level, thus may be increases feed intake and explained the highest body weight of birds fed on germinated seeds and this similar to that finding of Saford et al (1973) . The result was also on line with Makokkha et al (2002) who reported that germination increases the rate of minerals available, and soaking reducing phytic acid $43 \%$. The high growth obtained by birds fed on processed sorghum with vitamin $\mathrm{C}$ this could be explained by the fact that acidic anion complexes with the minerals like $\mathrm{Ca}$ and $\mathrm{P}$ result in an improvement in the digestibility of these mineral, ascorbic acid has $\mathrm{PH}$ reducing property and thus conducive for the growth ,the result in the same understanding of Siegenberg et al (1991) who reported that adding 60 $\mathrm{mg}$ of vitamin c counteracted phytic acid load of meal .In other study $80 \mathrm{mg}$ of ascorbic acid counteracted $2.5 \mathrm{mg}$ of phytic acid. Snow et al (2004) reported that addition of citric acid to broiler diets improve the tibia ash without reducing the weight gain or feed intake. Leg abnormality, serum Cholesterol, serum Glucose, and Tibia ash were the strong effect of reducing phytic acid from the poultry diets. The leg abnormality appear in birds fed in high amount of phytic acid than birds fed in low amount of phytic acid, this can be explained by the finding of Punna and Roland (1999) who reported that phytic acid reduced growth and caused leg abnormality as a sign of phosphorous deficiency . In the same direction (Atwal et al ,1980) reported that feeding rats with rape seed protein diets containing $1.24 \%$ phytate resulted in reduced growth rates and feed intake and efficiency of protein utilization of these animals . Moreover in early as 1949 the researcher Edward Mellanby, discovered that consumption of high -phytate cereal grain interfere with bone growth, and interrupts vitamin D metabolism resulted in rickets and a severe lack of bone formation (Mellanby, 1949), so most people had a diets high in phytate cause mineral deficiencies for example richest and osteoporosis are common in societies where cereal grain are staple part of the diet. The result (Table14) shown significant increase ( $p>0.05)$ in whole blood serum cholesterol obtained by birds fed on a high amount of phytate diet (in control), may be explained by the results of Sharon and Thomposon (1997) who reported that an addition of phytic acid to a high cholesterol diet reduced both serum cholesterol and triacyl glycerols . It is different from that obtained by Szkudelski(1998) who reported that phytic acid increased the cholesterol content in the whole blood serum in the rats .Result shown significant increase ( $p>0.05)$ in Calcium of whole blood serum obtained by birds fed on diet content a low amount of phytate, and shown decrease in phosphorous of whole blood serum ,may be explained by the finding of Sharon and Thompson (1997) who indicated that phytic acid reduced mineral bioavailability in both animal and humans. A similar observation was obtained by Punna and Ronald (1999). It is well known that the major phosphorus content of the grain is in the 
form of phytate, which has low available to monogastric animal, such as poultry (Simon ET al.1990: Summers, 1997. Bed ford2000,:Lesson and Summers ,2001) .phytic acid not only reduce phosphorus availability for poultry ,but also reduce the available of other nutrients in poultry ( Ravindran et al ,19999:Punna et al ,2001:Shirley and Edwards ,2003), so poor utilization of phytate phosphorus by monogastric poses several problems for producer.

Table15: Effect of feeding processed sorghum on broiler leg abnormality and blister (\%)

\begin{tabular}{|c|l|l|l|l|l|l|l|}
\hline \multicolumn{2}{|c|}{ Treatments } \\
\hline Parameters & $\begin{array}{c}\text { Unprocessed } \\
\text { sorghum } \\
\text { (control) }\end{array}$ & $\begin{array}{c}\text { Processed } \\
\text { sorghum } \\
\text { deulling }\end{array}$ & $\begin{array}{c}\text { processed } \\
\text { sorghum } \\
\text { (germination ) }\end{array}$ & $\begin{array}{c}\text { processed } \\
\text { sorghum( } \\
\text { soaking) }\end{array}$ & $\begin{array}{c}\text { processed } \\
\text { sorghum } \\
\text { (adding vita C) }\end{array}$ & $\begin{array}{c}\text { processed } \\
\text { sorghum } \\
\text { (storing) }\end{array}$ & $\begin{array}{c}\text { SE } \\
( \pm)\end{array}$ \\
\hline $\begin{array}{c}\text { Leg } \\
\text { abnormality }\end{array}$ & 4 & 0 & 0 & 0 & 0 & 0 & \\
\hline Blister & 2 & 0 & 2 & 2 & 0 & 2 & \\
\hline
\end{tabular}

Table16: Effect of feeding processed sorghum on Tibia Ash

\begin{tabular}{|c|c|c|c|c|c|c|c|}
\hline \multicolumn{10}{|c|}{ Treatments } \\
\hline $\begin{array}{c}\text { Para } \\
\text { meter } \\
\text { s }\end{array}$ & $\begin{array}{c}\text { Unprocessed } \\
\text { sorghum } \\
\text { (control) }\end{array}$ & $\begin{array}{c}\text { Processed } \\
\text { sorghum } \\
\text { (milling) }\end{array}$ & $\begin{array}{c}\text { processed } \\
\text { sorghum } \\
\text { (germination ) }\end{array}$ & $\begin{array}{c}\text { processed } \\
\text { sorghum } \\
\text { (soaking })\end{array}$ & $\begin{array}{c}\text { processed } \\
\text { sorghum } \\
\text { (adding vita C) }\end{array}$ & $\begin{array}{c}\text { Processed } \\
\text { sorghum } \\
\text { (storing ) }\end{array}$ & $\begin{array}{c}\text { SE } \\
( \pm)\end{array}$ \\
\hline $\begin{array}{l}\text { Tabia } \\
\text { Ash }\end{array}$ & $53.80 \pm 1.09 \mathrm{~b}$ & $\begin{array}{l}56.66 \pm 0.6 \\
1 \mathrm{a}\end{array}$ & $57.06 \pm 0.59 \mathrm{a}$ & $56.32 \pm 1.45$ & $57.14 \pm 0.48 \mathrm{a}$ & $\begin{array}{l}56.40 \\
\pm 1.70 \mathrm{a}\end{array}$ & $\begin{array}{l}0.4 \\
9\end{array}$ \\
\hline
\end{tabular}

*Means on the same row showing common superscripts are not significantly different at $0.05 \%$ level.

* SE: stander error of the means

\section{CONCLUSION AND RECOMMENDATION}

The results of this study showed that: Sorghum bicolor (Fetarita Gadarif) the main dish of poultry diet in Sudan had a high amount of phytic acid. The processing of sorghum using simple methods such as dehulling, soaking, germination, storing and adding of vitamin $\mathrm{C}$ had a good effect to reducing phytic acid in sorghum content. The finding of this study have shown some of the benefits attributed to the feeding of processed sorghum to the broiler chicks .Feeding processed seeds results in better performance of broiler chicks and increased protein and fat content of the carcass. These benefits are attributed to the low amount of phytic acid in processed seeds. Germination of seeds was the best method to reduced phytic acid, and this treatment obtained highest performance than the other good methods of experiment. Reducing of phytic acid using simple methods among seeds protect the stock from leg abnormality and richits. Processed sorghum low in phytic acid content reduced the cholesterol of whole blood serum. Processed sorghum low in phytic acid increased the broiler Tibia Ash and thus leads to a good health of the pone. Finally, in a addition of the above mentioned benefits of feeding processed grains, the methods of processing was also reduced the other anti nutritional factor such as Tannin and poly phenlics .

\section{REFERENCES}

[1] Abd Elnour , K.M .(200,).The Effect of decortications on wet milling and starch quality of sorghum and millet grain .M Sc, Thesis ,faculty of Agric. University of Khartoum, Sudan

[2] Abdelrahman, S.M.H.B, Elmakki, W.H .Idris, A.B.Hasan, W.E. Babiker andA.H.El-Yinay.2007. Antinutritionalfactor content and hydrochloric acid extractability of minerals in pearl millet cultivars by germination. Int. J, Food Sci Nutr, 58:6-17.

[3] Ahmed, E. F. (1993) Comparative study of wet milling properties of some sorghum varieties .Msc. Thesis, Faculty of Agric. University of Khartoum .Sudan

[4] AOAC. (1980) Official Methods of Analysis Association of Official Analytical Chemists Washington, DC.20044.po pox540.

[5] AOAC. (1995) Official Methods of Analysis Association of Official Analytical Chemists Washington, DC.

[6] Aotzc and Others .2001.Ahome-based method to reduce phytate content and increase zinc bioavailability in maize based complementary diets .International Journal of Food Sci. and Nutrition ,52:133-142 
[7] Atwal, A.S, Eskin, N.A.M.MC.Donald, B.EandVaisey-Genser, M, 1980.The effect of phytate on nitrogen utilization and zinc metabolism in young rates, Nutr. Rep. Int 21:257-267.

[8] Bedford, M.R.2000 .Exogenous enzymes in monogastric nutrition -their current value and future benefits Anim.Feed.Sci.Tec, 86:1-13.

[9] Chapman H D and Pratt F. P 1982 Determination of Mineral titration of analysis for soils plants and water .Edition Agriculture Division .California University U.S.A pp 169-170

[10] Chapman, B. M and Mahjan, L.1988 Effect of Natural Fermentation on the Extractability of minerals from pearl millet flour. J food Sic 53 1576-1577.

[11] Chapman, H. D and Peatt,F.P1961.Ammonium Vandate molybdate Method for Determination of Phosphorus .Methods of analysis for soils plants and water .Edition Agriculture Division .California University .U.S.A PP184=203

[12] Chapman, H.D, andPratt, F, P.1961.AmmoniumVandate-molybdate methods for Determination of Phosphorus, Methods of analysis for soil, plant and water. IEdition, Agriculture Division, California .University, U.S.A,pp,184-203

[13] Douglase, J. H, T.W, Sullvan, P. L. Bond and F.J.Strawem1990.Nutriient composition and metabolizable value of selected sorghum grains varieties and yellow corn in broiler diets. Poultry .Sci.69:1147-1155.

[14] Emmambux, M. N and Taylor, R.N (2009) Properties of heat -treat men ted sorghum and maize meal and their prolamin proteins .J .Agric, Food chem., 57, 1045-1050.

[15] Frings, Christopher. S. Dunn, MT. (1970) a colorimetric method for determination of total serum lipids based on the sulfo-phsphoro-vanillin reaction.American journal of clinical pathology volume 53, page 8991.

[16] Greiner R. Konietznyu (2005) Phytase for food Application .Food Technol Biotechnol: 44, 125-140.

[17] Harland, S.C.1936. The genetical conception of the species .Biolodical Review 11:83-112.

[18] Hulse.J.H:Laing.E.M and Pearson, .E (1980). Sorghum Millets: their composition and nutritive value.NewYork.Acadimic Press997p

[19] Idris, W.H (2004) Effect of processing on the anti-nutritional factors and mineralsbioavailabiliyofsorghum.PhD.Thesis.Faculty of Agric, University of Khartoum.Sudan.

[20] Kim, E. and Goldbig.M (1969). Serum cholesterol assay using astable Lieberman-Burchard reagent.Clinical. chem...15 (12)117-9

[21] Kouakou,B.K,K,S,Alens,D.Alexis,D.Adjehi,D,K.Marcelin and G.Dago (2008).Biochemical changes occurring during germination and fermentation of millet and effect of technological processes on starch hydrolysis by the crude enzymatic extract of millet. J. Applied .Sci,RES 4:1502-1510

[22] Lesson, S, and J.D.Summers, 2001.Nutrition of the chickens, 4th Edition pp, 331-428(University books.P.O.Box 1326, Guelph.ontario, Canada. NIH 6NB)

[23] Mahgoub, S.E.O and S.A.Elhage, 1998.Effect of milling soaking, malting heat treatment and fermentation on phytate level of flour Sudanese sorghum cultivars .food chem...61:77-80.

[24] Makokha,A.O,Oniango,R.K,Njoroges.M,Kamaraok, (2002).Effect of traditional fermentation and malting on phytic acid and mineral availability from sorghum and finger millet grain varietirs grown in Kenya.University of Agriculture and Technology ,department of food science.Journal Articil, Research Support-Non-U.S Govt

[25] Mamudu.H.B, Jideani and + I .Nkama, 2005 .Sugar content of pereal millet as diversed amount cultivars and affected by germination. J.Applied Glycosci, 52:331-335.

[26] Marero ,L.M,Payumo,E.M.,Aguinaldo, A.R.,Mastsumoto,I,and Homma,S.19 .Anttinutrtional factor in weaning foods prepared from germinated cereals and legumes .Lebensm .Wiss.U. Technol. 24:177-181.

[27] Marfo.E.KSimpson.B.K Idowu,JS.andOke.O.L (1990)Effect of local food processing of phytate level in cassava,cocoyam,yam,maize, sorghum,rice and soybeen.J.Agric.Food Chem.38:1580-1585

[28] Martinez,A.B,R.K,Simposon,J.S.Idwu andR,Bressan,1980.Relation of lysine and tryptophan tothat of Z,during germinationof grain of corn and it is possible linkage with the growing cycle of theplant.Aresearch, Latinoam,Nutr,30:607-633.

[29] Mellanby, E.1949.The Rickets-producing and ant-calcifying action of phytate.Journal of physiology, 109:pp488-533.

[30] Mullaney,Edward.J:Ullah,Abul.H.J: (2012) Phytaseatrributes-catalytic mechanisms and application .United State Department of Agriculture-.Agricultural Research Service .Retrieved May 18.2012 
[31] MyadaAliasMustfa(2009).Evaluation of nutrients content of sorghum flour supplemented with wheat and pumpkin seed flour. MSc thesis. Faculty of Agriculture University of Khartoum Sudan

[32] NRC. (1984) National Research Council. Nutrient Requirement of poultry , $8^{\text {th }}$,rev. ed . Washington. DC .National Academy. Sci. Press

[33] Ockenden,.I,Falk,D.E. and Lott,J.N.A.1997.Stability of phytate in barley and beans during storage. J .Agric.Food Chem.45:1673-1677.

[34] Ocheme,O.B,andC.E.Chinma, 2008. Effect of soaking and germination on some physicochemical properties millet flour for porridgeproduction.J .Food Technol, 6:185-188.

[35] Omar.E.Omara (2000).Effect of feeding whole millet grain on broilers performance. MSc thesis .Faculty of Animal Production. University of Khartoum Sudan

[36] Osbbome, D. R and Voogt, P 1978. Calculation of caloric value In Analysis of Nutrients in foods Academic Press New York pp 23-34

[37] Punna.s, and Roland.Sr.1999. Variation in phytate phosphorus utilization within the same broilers strain .J.App.Poultry Research, volume 8, issue 1.page 10=15.

[38] Ravindran, V.S.Cabahug, G.Ravidran and W.L.Bryden .1999b.Influnce of microbial phytase on apparent ileal amino acid digestibility of feed stuffs for broilers. Poult.Sci.78:699-706.

[39] Rose, A.R.1912.A resume of the literature on inosite-phosphoric acid with special reference to the relation of that substanceto plants .Biochem.Bull.2:21-49.

[40] Sathe.N.Rukma Reddy Shridhark.( 2002).Food Phytates.International Stander Book Number 1-5,66768675,CRCPress,Boca Raton London New York Washington,D.C.

[41] Savanberg U and Lorri.W19987Fermintationand Nutrition Availability .food cont-8 319-327.

[42] Sharon,E.Ricard and Lilian,U. Thompossn. 1997. Interaction and Biological effect of phytic acid. Department of Nutrition Sci.Faculty of Medicin.University of Tornto,ontarjo MSS 3E2.Canada.

[43] Shirly,R.B and H.M.Edward,Jr.,2003.Graded levelsof phytase past industry standard improves broiler performance .Poult .Sci.82:671-680.

[44] Siegenberg D, Baynes RD,Bothwell TH, Macfarlane BJ, Lamparelli RD ,Car NG, Macphail p, Schmidt U, Tal A,Mayet F . (1991). Ascorbic Acid prevents the dose dependent inhibitory effects of polyphenols and phytates on nonheme- iron absorption. Am. J .Clin .Nutr, Feb: 53(2):537-41.

[45] Snedecor,G.W and W,G. Cochran.(1987) .Statistical Methods"Printing,.The lawa State . University Press, Amcs, Jowa, U.S.A.

[46] Snow,J.L.,D.H.Baker, and C.M.Parsons. 2004 Phytase citric acid and 1-hydroxycholecalci ferol improve phytate phosphorus utilization in chicks fed acorn-soybean meal diet. Poultry Sci, vol 83, no 7 pp $1187=1192$

[47] Summers, J.D.1997. Precisio phosphorus nutrition..J.Appl .Poult, Res, 6:495-500.

[48] Trinder.P. (1960). Colorimetric micro determination of Calcium in serum analyst 85:889-894.

[49] T.S.Nelson (1967).The utilization of phytate - phosphorus by poultry: a review- poultry sci.46 pp862-871.

[50] Valencia,S, Svanberg, U,.Sandberg, A,S and Ruales, J. 1999. Processing of quinoa (Chenopodium quinoa,Wild): effects onin vitro iron availability and phytate hydrolysis. Int. J .Food Sci, Nutr.50:203-211.

[51] Wheeler,E.L and Ferrel,R.E (1971) Amethod for Phytic Acid Determination in wheat and wheat fractions .Cereal Chemistry,48,312-320

[52] WHO.1998. Complementary of young children in developing countries Areview of current scientific knowledge WHO, Geneva, http://apps.who.int/bookorders/anglais/detart1.jsp session=1\&codlan= $1 \& \operatorname{codco} 1=93 \& \operatorname{cod} c \mathrm{ch}=141$

[53] Wisal Hasan Idris Badi Effect of processing on Anti-Nutrition Facter and minerals Bioavailability of Sorghum .Faculty of Agric University of Khartoum.Sudan

Citation: O. E. Omara Ahmed, "Effect of Reducing Phytic Acid from Sudanese Sorghum Bicolor (F.G) Using Simple Technique Methods on Broilers Performance ", International Journal of Research Studies in Agricultural Sciences, vol. 3, no. 12, p. 18-30, 2017. http://dx.doi.org/10.20431/2454-6224.0312003

Copyright: (C) 2017 Authors. This is an open-access article distributed under the terms of the Creative Commons Attribution License, which permits unrestricted use, distribution, and reproduction in any medium, provided the original author and source are credited. 